\title{
Design and Experimental Evaluation of a Tendon-Driven Minimally Invasive Surgical Robotic Tool with Antagonistic Control
}

\author{
Nikolaos Evangeliou ${ }^{\dagger}$, Emmanouil Dimitrakakis ${ }^{\dagger}$, and Anthony Tzes ${ }^{\ddagger}$
}

\begin{abstract}
The design, implementation and experimental evaluation of a minimally invasive surgical robotic instrument is presented in this article. The tool is constructed using rapid prototyping techniques and each degree-of-freedom is actuated via an antagonistic tendon driven mechanism using servo motors. The accompanying software runs under the Robot Operating System framework. The kinematics of the tool are discussed and the efficiency of the system is investigated in experimental studies, which are showcased in order to assess its potential use in a clinical environment.
\end{abstract}

\section{INTRODUCTION}

In recent years, research in robot-assisted Minimally Invasive Surgery (MIS) has been exponentially rising. The faster recovery time, reduced pain, reduced discomfort and lower risk of infection has lead to an increased demand for robotic systems that aid in MIS operations [1]. Thus, several prototypes [2]-[7] and commercial systems [8], [9] have appeared.

Most MIS-robots are actuated by DC-motors due to their ease of control and integration, as well as their ability to exert large torques. Despite the lower power-to-weight ratio of motors compared to shape-memory-alloy or pneumatic actuators [10]-[12], motors remain the preferred means of actuation in surgical robotics.

The actuation system is always placed outside the operational cavity, proximally to the active joint, in order to isolate and miniaturize the robot [13]-[15]. The antagonistic control scheme that is usually applied is inspired by the physiological structure of the human joint in a puller-follower antagonistic configuration. There is a clear distinction between the pulleragonist motor, that is responsible for the motion of the joint, and the follower-antagonist whose main responsibility is preventing the tendon from becoming slack [16]. Antagonistic control, with simple control-strategies can offer a fast transient response, yet when accuracy and repetitiveness are required, the necessitated control strategies are quite sophisticated [17]-[20].

In this paper a two Degree-of-Freedom (DoF) robotic probe for MIS operations is designed and fabricated. Its kinodynamic model is extended for an $N$ DoF tendon-driven manipulator. The physical prototype uses servo motors with embedded PID control scheme for actuation. Experimental

This work was funded by the IKY Fellowships of Excellence for Postgraduate Studies in Greece: Siemens Program

$\dagger$ Evangeliou and Dimitrakakis are with the Electrical \& Computer Engineering Department University of Patras, Rio, 26500, Greece.

$\$$ A. Tzes is with the Engineering Division, New York University Abu Dhabi, United Arab Emirates. Corresponding author's email: anthony.tzesenyu.edu results are presented to evaluate the robotic probe's feasibility to move under variable payloads in an antagonistic fashion.

The main novelties of the implementation are its low weight and size making the robot suitable for surgical operations. Moreover, the probe can carry a large payload, while its rapid prototyping implementation allows for interchangable modules, thus on-the-fly modular design.

The paper is organized as follows: in Section II, the design and fabrication of the surgical instrument is presented. The kinematic analysis for a generic $N$ DoF robotic tool of the same configuration is showcased in Section III and further specified for the physical prototype where $N=2$. In Section IV preliminary experimental results are presented followed by the conclusions Section in V.

\section{Design of the MIS-Robotic ToOL}

The 2-DoF MIS-robotic tool was designed and fabricated by cascading identical dual links and joints in a tendondriven configuration. The joints/links as well as the motors' fixation base are manufactured using rapid prototyping techniques with $A B S$ plastic. Insertion is achieved through a T6065 aluminum hollow shaft allowing for safe tendon guiding. The use of rapid prototyping techniques allows for fast alteration of the distal end's design since quick fabrication of each new configuration per physician request is feasible. Thus, the system can be characterized as an increased modularity system, provided that the operational needs and consequently robot's design parameters are preoperatively defined.

The outer diameter of the robotic probe, shown in Fig. 1, is $15 \mathrm{~mm}$ making it suitable for MIS operations. The resulting rotational DoFs have their axes of rotation placed perpendicular with respect to each other. A shaft of $15 \mathrm{~mm}$ in diameter is used for insertion of the instrument to the operating cavity and a $3 \mathrm{~mm}$ middle working channel is also utilized to allow for catheterization. Four channels are created at a perimeter of $5 \mathrm{~mm}$ from the center of each joint and link, utilizing tendon passages. An antagonistic tendon pair passing diametrically through the aforementioned channels is used to actuate each DoF. The selected tendons are steel wires with a $7 \times 7$ strand configuration, a diameter of $0.5 \mathrm{~mm}$ and a tensile strength limit of $750 \mathrm{~N}$.

The actuators used in this work are high efficiency Dynamixel servo-motors [21], with a stall torque of $28 \mathrm{~kg} \cdot \mathrm{cm}$, an angular step resolution of $0.088^{\circ}$ and an embedded PID position control scheme. Communication is achieved through a single USB-to-RS485 bus at a rate of $50 \mathrm{~Hz}$. The overall weight of the MIS probe is $0.65 \mathrm{Kg}$. The tendons are fixed to 


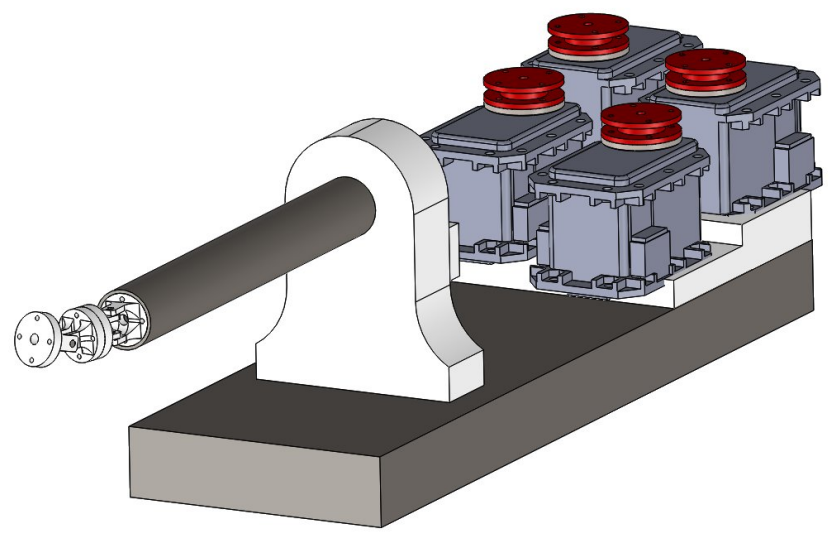

Fig. 1: 2-DoF MIS robotic tool CAD

their corresponding motors at a radius of $R_{p}=6.5 \mathrm{~mm}$ using a custom fabricated pulley, as shown in Fig. 2.

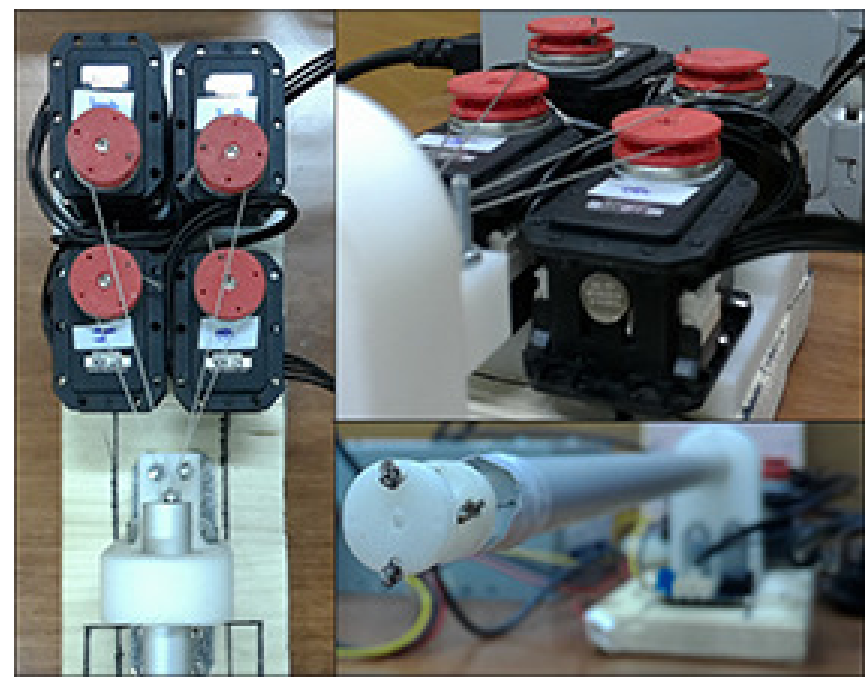

Fig. 2: MIS-robotic tool prototype

\section{KInematic ANALYSIS OF THE MIS-Robotic TOOL}

The Denavit-Hartenberg (DH) kinematic parameters for an $N$-DoF robotic manipulator integrating the adopted configuration are shown in Table $\mathrm{I}$, where $i=1, \ldots, N$. In the presented design, $\theta_{i} \in\left[-50^{\circ}, 50^{\circ}\right], L_{i}=L_{i+1}=20 \mathrm{~mm}$.

\begin{tabular}{|c|c|c|c|c|}
\hline Link & $\theta_{i}$ & $\mathrm{a}_{i}$ & $\alpha_{i}$ & $d_{i}$ \\
\hline$i$ & $\theta_{i}$ & $L_{i}$ & $90^{\circ}$ & 0 \\
\hline$i+1$ & $\theta_{i+1}$ & $L_{i+1}$ & $-90^{\circ}$ & 0 \\
\hline
\end{tabular}

TABLE I DH parameters for 2 robot's consecutive joints

In our case, where $N=2$ the homogenous transformation matrix $A_{0}^{2}$ expressing the tip's pose can be expressed as in Eq. 1, where $s_{i}=\sin \left(\theta_{i}\right), c_{i}=\cos \left(\theta_{i}\right), i=1,2$ and $l=10 \mathrm{~mm}$. Consequently, the resulting workspace is depicted in Fig. 3.

$$
\begin{aligned}
A_{0}^{2} & =\left[\begin{array}{ccc|c}
c_{1} c_{2} & -c_{1} s_{2} & -s_{1} & l\left(2 c_{1}+c_{1} c_{2}+1\right) \\
s_{2} & c_{2} & 0 & l s_{2} \\
c_{2} s_{1} & -s_{1} s_{2} & -c_{1} & l\left(2 s_{1}+s_{1} c_{2}\right) \\
\hline 0 & 0 & 0 & 1
\end{array}\right] \\
& =\left[\begin{array}{c|c}
R_{0}^{2} & P_{0}^{2} \\
\hline 0_{1 \times 3} & 1
\end{array}\right]
\end{aligned}
$$

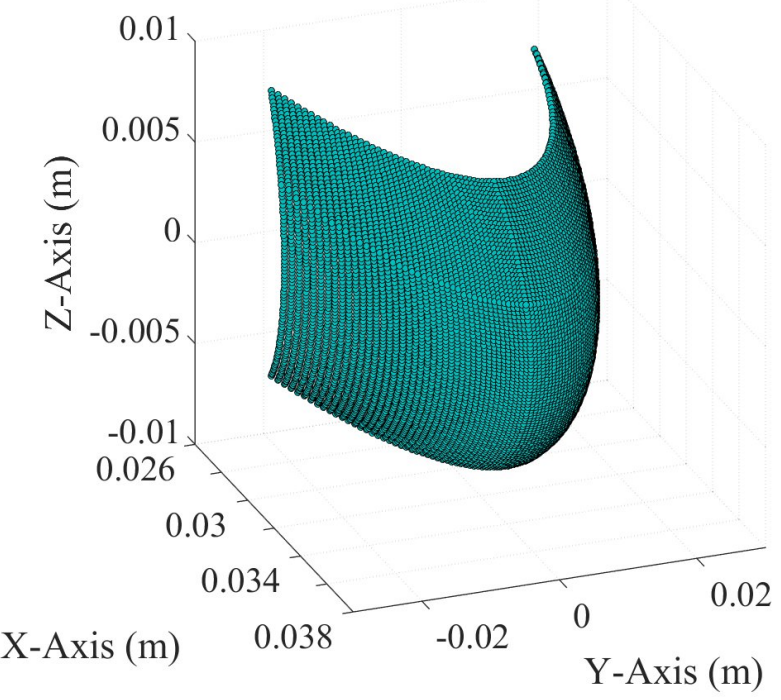

Fig. 3: 2DoF MIS-robotic tool workspace

The inverse kinematics position problem for a redundant $N$-DoF manipulator is overdetermined with infinite number of candidate solutions. Thus, a numerical approach was implemented for the calculation of the angle-vector $\theta\left(t^{+}\right)=\left[\theta_{1}^{+}, \ldots, \theta_{N}^{+}\right]^{T}$ of the robot's DoF at time $t^{+}$. Given the current angles $\theta\left(t^{-}\right)=\left[\theta_{1}^{-}, \ldots, \theta_{N}^{-}\right]^{T}$ at time $t^{-}$and the desired position vector of the end-effector $P_{0}^{N, d}\left(t^{+}\right)=$ $\left[P_{x}^{d}\left(t^{+}\right), P_{y}^{d}\left(t^{+}\right), P_{z}^{d}\left(t^{+}\right)\right]^{T}$ at time $t^{+}$the computation is cast as a Sequential Quadratic problem ( [22]) defined in Eq. 2.

$$
\min _{\theta}\left\|\theta\left(t^{+}\right)-\theta\left(t^{-}\right)\right\|
$$

$$
\text { subject to }\left\|P_{0}^{N, d}\left(t^{+}\right)-P_{0}^{N}\right\|=0,
$$

where $P_{0}^{N}$ the resulting end-effector's position, as shown in Fig. 1 for $N=2$. In a typical experiment $t^{+}-t^{-}$corresponds to the sampling period $T_{s}$.

Assuming a computed joint space angle-vector, the tendon stroke of each antagonistic pair must be computed for positioning precision. As previously described, for each rotational DoF there are two tendons running from the robot's base, passing through holes at each joint and ending at the counter-diameter holes at the corresponding joint to be rotated. To compute the tendons' lengths, it is crucial to compute the length of the path of each tendon, which 
necessitates the computation of the locations of the passingthrough holes in $3 D$ space.

For an $N$ DoF robotic tool, each link has $2 N$ symmetrically positioned channels at a radius $r$ from its center of symmetry; the angle on the joint's plane between each hole is $\frac{360^{\circ}}{2 N}$. The computation of the tendon's path length is crucial in the antagonistic control and is accurately computed by expressing each hole in $3 D$ space and using the resulting homogenous transformation matrix per hole and Euclidean geometric calculations. As an example, for a 10-DoF robot in a random positioned configuration, the tendon and joint configuration are depicted in Fig. 4 in black and red color respectively.

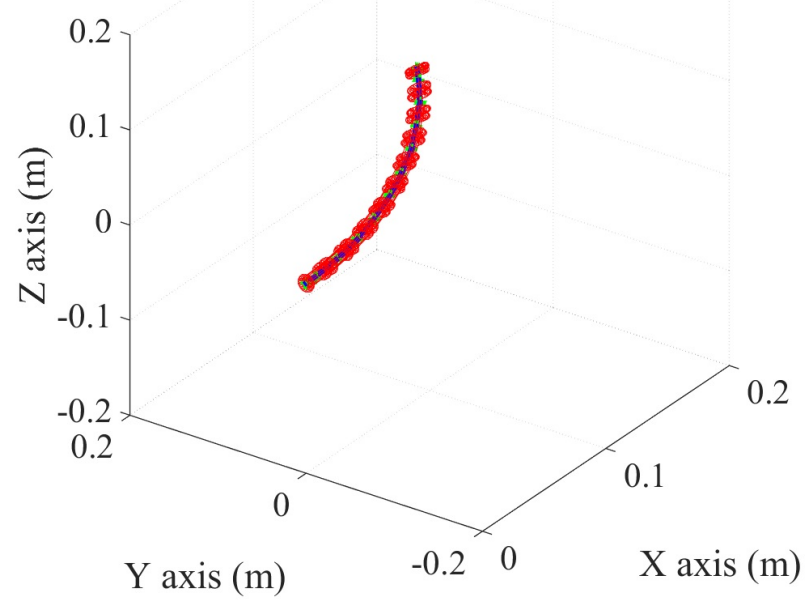

Fig. 4: Tendons' pose in 3D-space for a $10-\mathrm{DoF}$ robotic tool configuration

\section{EXPERIMENTAL STUDIES}

The open-source experimentation software of the robot was developed under the Ubuntu Linux operating system utilizing the ROS framework [23]. The later allows for fast integration of sensors and software modularity. Specifically, two nodes were implemented so as to compute the rotation angles per user input and translate them to control signals and to communicate with the motors respectively.

The carrying payload feasibility of the robot was evaluated experimentally by mounting incrementally increasing deadweights on the end effector. It was visibly confirmed that the robot can carry up to $380 \mathrm{~g}$ and move to its angular extremities without significant deviations, while its terminal failure point occurred for a weight of $620 \mathrm{~g}$. Subsequently, a simple yet effective antagonistic actuation scheme was defined so as to avoid slackness and dead-zone during tissue interaction. Specifically, based on the nominal weight of $380 \mathrm{~g}$, a lead time of $4 \mathrm{~ms}$ to the puller actuation was experimentally found to produce repetitive results with no slackness.
The efficiency of the MIS robotic tool for autonomous surgical tasks was evaluated by comparing the position feedback from the motor's encoders to the measurements of an IMU sensor. Initially the robot is commanded to move from the position defined by the DH parameters $\theta_{1}=\theta_{2}=-30^{\circ}$ to the position defined by the DH parameters $\theta_{1}^{d}=\theta_{2}^{d}=30^{\circ}$. The response of each motor is shown in Fig. 5.
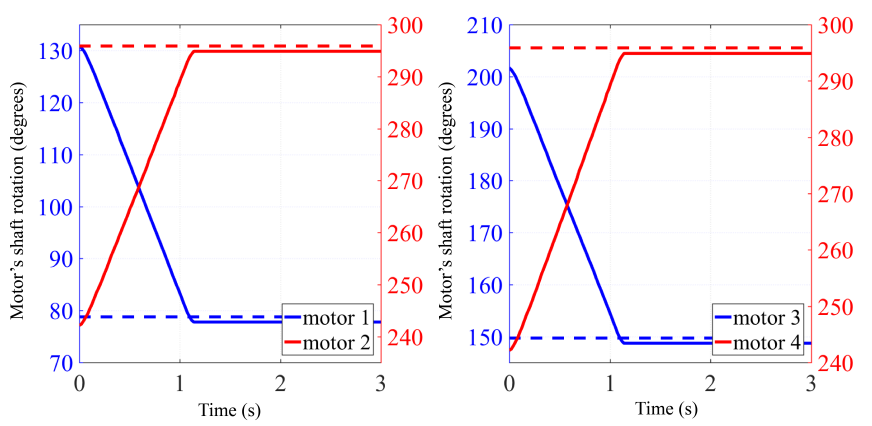

Fig. 5: Antagonistic motor response

The system's response was evaluated using a 9-DoF IMU sensor attached at the tip of the robot. In Fig. 6 a comparative graph of the end-effector's pose, using the two different sensing systems, is depicted. Specifically, the blue line indicates the IMU measurement and the red line indicates the position of the end-effector based on the feedback from the motor's encoders.

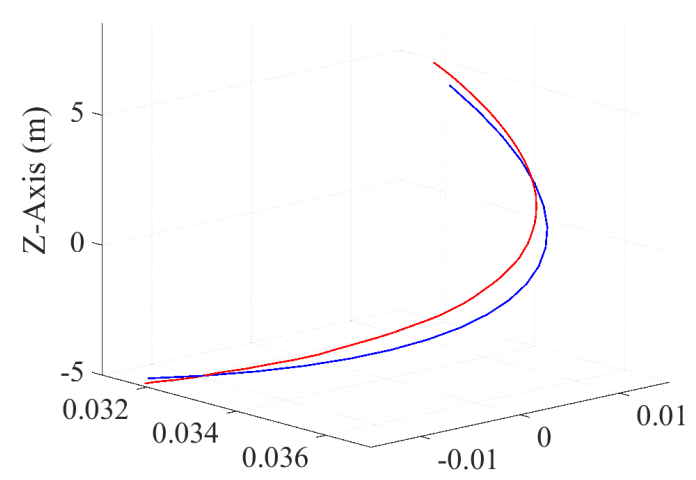

Fig. 6: Comparative end-effector's position for single motion command

An additional experimental procedure was the circular path following case. Let assume a predefined circular motion of radius $r$ and $L$ the distance between the plane of the end effector for $\theta_{1}=\theta_{2}=0^{\circ}$ and the plane of the circular path. A total of 20 points is generated on the circumference of the circle with center $(L, 0,0)$ and radius $r$, assuming that the initial position of the end effector is at $(0,0,0)$. Then, the equation of the line between the end effector position and the position of the second DoF can be computed in a parametric manner as a function of $\theta_{1}$ and $\theta_{2}$. The corresponding mathematical notation is depicted in Eq. 3.

$$
\langle x, y, z\rangle=\left\langle x_{0}, y_{0}, z_{0}\right\rangle+t\left\langle m_{x}, m_{y}, m_{z}\right\rangle
$$


where $t$ is the points of the line iterator and $m$ is the slope of the line. In our case Eq. 4 and Eq. 5 hold, where $P$ the position of the end-effector and $P_{D}$ the position of the second DoF using the forward kinematics analysis.

$$
\left\langle x_{0}, y_{0}, z_{0}\right\rangle=\left\langle P_{x}, P_{y}, P_{z}\right\rangle
$$

and

$$
\left\langle m_{x}, m_{y}, m_{z}\right\rangle=\left\langle P_{x}-P_{D x}, P_{y}-P_{D y}, P_{z}-P_{D z}\right\rangle
$$

The positions that the end effector must reach in order for the laser to track the $c_{i}$ points on the desired circle are computed by solving the system of Eq. 6 w.r.t. $\left[t, \theta_{1}, \theta_{2}\right]$.

$$
\left\langle c_{i x}, c_{i y}, c_{i z}\right\rangle=\langle x, y, z\rangle
$$

The points on the desired circle, alongside the poses that the robot must reach and the lines that were computed for $r=7 \mathrm{~cm}$ and $L=15 \mathrm{~cm}$ are shown in Fig. 7 .

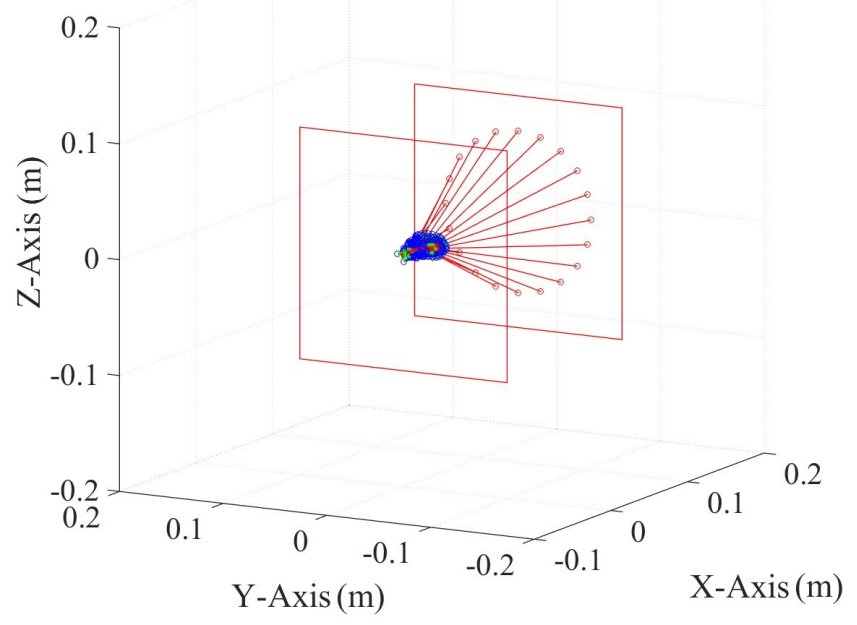

Fig. 7: Robot poses for a circle of $r=7 \mathrm{~cm}$ and $L=15 \mathrm{~cm}$

The robotic instrument is commanded to autonomously follow the specified circular path and using long exposure photography techniques the resulting path as compared to the reference is shown in Fig. 8. The visible low tracking error is mainly attributed to the improved feedback accuracy of the integrated servo-motors.

The time response of the positions of the motors for this particular circular path are displayed in Fig. 9.

\section{CONCLUSIONS}

A 2 DoF light-weight and compact robotic instrument was designed, constructed and evaluated for tissue interaction during robot-assisted MIS interventions. Each joint is actuated via control of the lengths of antagonistic tendons, with the use of high efficiency servo motors. The maximum angle of rotation error of $0.2^{\circ}$ per DoF as shown in Fig. 5, the low overshooting error in Fig. 9 and the low error of $1 \mathrm{~mm}$ in the final position of the end effector depicted in Fig. 6 encourage the exploitation of clinical scenarios, as

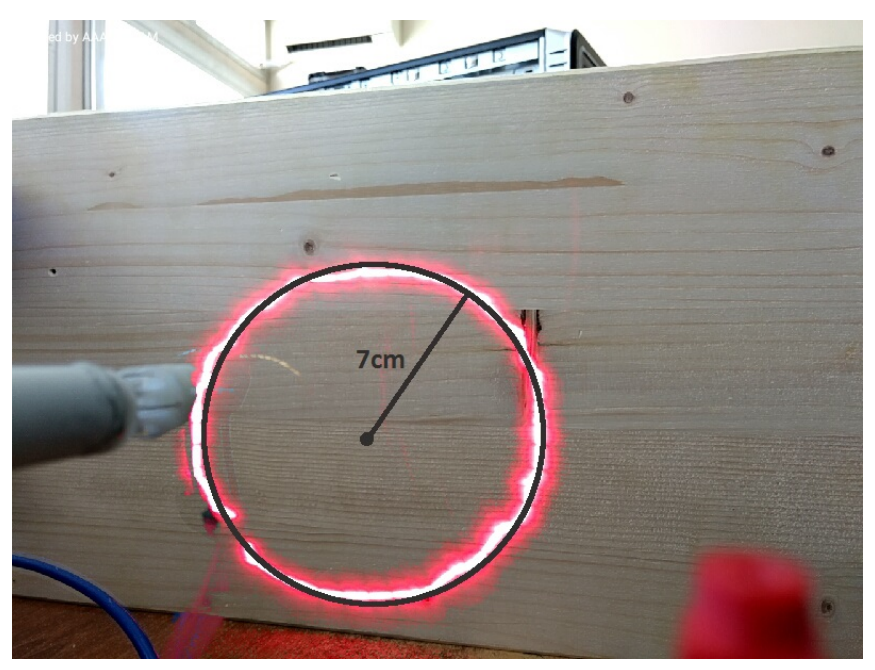

Fig. 8: Circular path with $r=7 \mathrm{~cm}$ and $L=15 \mathrm{~cm}$
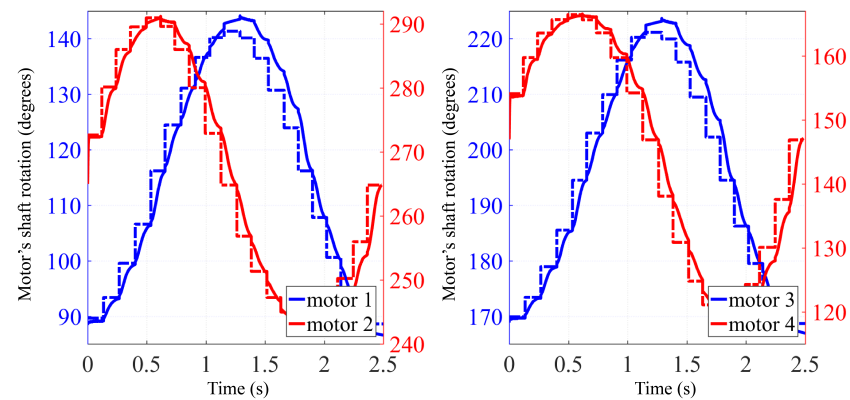

Fig. 9: Motors' time response for circular path

well as advanced antagonistic control schemes for improved efficiency.

\section{REFERENCES}

[1] P. Gomes, "Surgical robotics: Reviewing the past, analysing the present, imagining the future," Robotics and Computer-Integrated Manufacturing, vol. 27, no. 2, pp. 261 - 266, 2011, translational Research Where Engineering Meets Medicine.

[2] N. Evangeliou and A. Tzes, "Development of an sma-actuated redundant robotic platform for minimally invasive surgery," in Biomedical Robotics and Biomechatronics (BioRob), 2016 6th IEEE International Conference on. IEEE, 2016, pp. 353-358.

[3] V. Vitiello, S. L. Lee, T. P. Cundy, and G. Z. Yang, "Emerging robotic platforms for minimally invasive surgery," IEEE Reviews in Biomedical Engineering, vol. 6, pp. 111-126, 2013.

[4] R. Konietschke, U. Hagn, M. Nickl, S. Jorg, A. Tobergte, G. Passig, U. Seibold, L. Le-Tien, B. Kubler, M. Groger, F. Frohlich, C. Rink, A. Albu-Schaffer, M. Grebenstein, T. Ortmaier, and G. Hirzinger, "The dlr mirosurge - a robotic system for surgery," in 2009 IEEE International Conference on Robotics and Automation, May 2009, pp. $1589-1590$.

[5] M. J. H. Lum, D. C. W. Friedman, G. Sankaranarayanan, H. King, K. Fodero, R. Leuschke, B. Hannaford, J. Rosen, and M. N. Sinanan, "The raven: Design and validation of a telesurgery system," The International Journal of Robotics Research, vol. 28, no. 9, pp. 1183$1197,2009$.

[6] U. Seibold, B. Kubler, and G. Hirzinger, "Prototype of instrument for minimally invasive surgery with 6-axis force sensing capability," in Proceedings of the 2005 IEEE International Conference on Robotics and Automation, April 2005, pp. 496-501.

[7] S. Eslami, G. S. Fischer, S. E. Song, J. Tokuda, N. Hata, C. M. Tempany, and I. Iordachita, "Towards clinically optimized mri-guided surgical manipulator for minimally invasive prostate percutaneous 
interventions: constructive design," in 2013 IEEE International Conference on Robotics and Automation, May 2013, pp. 1228-1233.

[8] M. T. Gettman, R. Peschel, R. Neururer, and G. Bartsch, "A comparison of laparoscopic pyeloplasty performed with the davinci robotic system versus standard laparoscopic techniques: Initial clinical results," European Urology, vol. 42, no. 5, pp. 453 - 458, 2002.

[9] L. van den Bedem, R. Hendrix, N. Rosielle, M. Steinbuch, and H. Nijmeijer, "Design of a minimally invasive surgical teleoperated master-slave system with haptic feedback," in 2009 International Conference on Mechatronics and Automation, Aug 2009, pp. 60-65.

[10] C. Mavroidis, C. Pfeiffer, and M. Mosley, "Conventional actuators, shape memory alloys, and electrorheological fluids," 1999.

[11] E. Tzorakoleftherakis, A. Mavrommati, and A. Tzes, "Design and implementation of a binary redundant manipulator with cascaded modules," Journal of Mechanisms and Robotics, vol. 8, no. 1, p. 011002, 2016.

[12] N. Evangeliou and A. Tzes, "Development of an sma-actuated redundant robotic platform for minimally invasive surgery," in 2016 6th IEEE International Conference on Biomedical Robotics and Biomechatronics (BioRob), June 2016, pp. 353-358.

[13] N. Evangeliou, D. Karageorgos, and A. Tzes, "Design and antagonistic control of a tendon-driven minimally invasive surgical robotic tool," in 2016 24th Mediterranean Conference on Control and Automation (MED), June 2016, pp. 725-730.

[14] Z. Li, L. Wu, H. Ren, and H. Yu, "Kinematic comparison of surgical tendon-driven manipulators and concentric tube manipulators," Mechanism and Machine Theory, vol. 107, pp. 148 - 165, 2017.
[15] S. C. Jacobsen, H. Ko, E. K. Iversen, and C. C. Davis, "Antagonistic control of a tendon driven manipulator," in Proceedings, 1989 International Conference on Robotics and Automation, vol. 3, May 1989, pp. 1334-1339.

[16] K. Koganezawa, G. Takami, and M. Watanabe, "Antagonistic control of multi-dof joint," in 2012 IEEE/RSJ International Conference on Intelligent Robots and Systems, Oct 2012, pp. 2895-2900.

[17] S. C. Jacobsen, H. Ko, E. K. Iversen, and C. C. Davis, "Control strategies for tendon-driven manipulators," IEEE Control Systems Magazine, vol. 10, no. 2, pp. 23-28, Feb 1990.

[18] N. Abroug and E. Laroche, "Transforming series elastic actuators into variable stiffness actuators thanks to structured $\mathrm{h}$ x221e; control," in 2015 European Control Conference (ECC), July 2015, pp. 734-740.

[19] B. Okur, O. Aksoy, E. Zergeroglu, and E. Tatlicioglu, "Nonlinear robust control of tendon-driven robot manipulators," Journal of Intelligent \& Robotic Systems, vol. 80, no. 1, pp. 3-14, 2015.

[20] V. Potkonjak, B. Svetozarevic, K. Jovanovic, and O. Holland, "The puller-follower control of compliant and noncompliant antagonistic tendon drives in robotic systems," International Journal of Advanced Robotic Systems, vol. 8, no. 5, p. 69, 2011.

[21] C. N. Thai, "Robotisrobot systems," in Exploring Robotics with ROBOTIS Systems. Springer, 2015, pp. 5-17.

[22] J. J. Moré and D. C. Sorensen, "Computing a trust region step," SIAM Journal on Scientific and Statistical Computing, vol. 4, no. 3, pp. 553$572,1983$.

[23] "ROS - Robot Operating System,” [Online], Available: http://www.ros. org/. 